\title{
Load flow analysis using decoupled fuzzy load flow under critical conditions
}

\author{
P. Acharjee ${ }^{1 *}$, Kawsar Ali ${ }^{2}$ \\ ${ }^{1 *}$ Department of Electrical Engineering, National Institute of Technology Durgapur, INDIA \\ 2 Department of Electrical Engineering, National Institute of Technology Durgapur, INDIA \\ "Corresponding Author: e-mail: parimal.acharjee@gmail.com,Tel+91-9231334093
}

\begin{abstract}
The conventional load flow methods like Newton-Raphson load flow (NRLF), Fast Decoupled load flow (FDLF) provide poor performance under critical conditions such as high R/X ratio, heavily loading condition etc. Exploiting the decoupling properties of power system, reliable fuzzy load flow is developed to overcome the limitations of the popular methods. Both power mismatch and summation of power mismatches are taken as two inputs for fuzzy logic controller. Considering magnitude of power mismatch and sign of power mismatch, new 25 fuzzy rules are created from two sets of inputs. The proposed Decoupled Fuzzy Load Flow (FDLF) is applied to IEEE 14 bus, IEEE 30 bus, IEEE 57 bus and IEEE 118 bus under stressed situations. The test results prove that the proposed algorithm provides better performance than the conventional methods under critical conditions.
\end{abstract}

Keywords: Load Flow, Decoupling Properties, Fuzzy Logic, Critical conditions.

\section{Introduction}

One of the most basic and commonly encountered problems of power system analysis is the load flow. Load flow has a rich tradition of research and as a result there are many efficient solution techniques of the load flow problem besides the general purpose solution techniques like the Newton-Raphson (Tinney et al, 1967) and the Fast Decoupled (Wang et al, 2000) methods. Occasionally, load flow suffers from the problem of convergence difficulties. Methods based on optimal multiplier power flow (OMPF) (Iwamoto et al, 1981) have been developed to overcome such situations. The requirements of the speed and accuracy of the load flow algorithm vary. Solution of the load flow problem in stressed condition, particularly when the system is operating at its loadability limit, is of great importance for voltage stability analysis. Conventional power flow methods fail to find a solution in such cases (Wong et al, 2003). Moreover, due to the introduction of FACTS based control devices (Lo et al, 1997) and the operating environment due to the restructuring of the power system, necessity for the development of rugged power flow algorithms utilizing the general purpose evolutionary techniques have been felt (Vlachogiannis, 2001).

The justification of the application of the non conventional approaches such as Genetic algorithm (GA), Particle Swarm Optimization (PSO), Fuzzy logic (FL) in the power flow problem lies in the fact that these approaches are not based on the power flow derivatives. So, these methods need not face the problem of Jacobian singularity as the maximum loading conditions are approached. GA and PSO based load flow algorithms are evolutionary based and stochastic in nature. But the second family of load flow algorithms incorporating uncertainty has been developed more recently and it utilizes fuzzy sets for its modeling (Miranda et al, 1998). This is qualitatively different way of expressing uncertainty. It represents imprecise, or vague, knowledge rather than uncertainty related to a frequency of occurrence. One inherent advantage of this approach is the ability to easily incorporate expert knowledge about the systems under study. With this approach, input variables are represented as fuzzy numbers, which are special types of fuzzy sets. Although the calculations in fuzzy analysis are somewhat simpler than that in a probabilistic case, it is still far too complex to be applied directly to the full system model. Therefore, again a linearized model of the system is used. Fuzzy interval arithmetic has been employed in handling uncertainties associated with the load, generation or system parameters. 
Fuzzy load flow is developed exploiting the decoupling properties of power systems. The maximum power mismatch and summation of power mismatches are taken as two input ranges. Two separate loops are used like fast decoupled power flows. To show the robustness, the proposed algorithm is applied to different IEEE test systems under critical conditions.

\section{Load Flow Problem}

The problem in load flow is to determine the voltages at (n-g-1) nodes and phase angles of the voltages at (n-1) nodes when the real power at $(n-1)$ nodes and the reactive power at $(n-g-1)$ nodes are specified. The reactive power limits of the $g$ number of generator nodes and the active power balances at all the nodes are to be satisfied. Since the number of variables goes on increasing with the size of the system, the load flow problem becomes increasingly difficult as the size of the system increases. If maintaining the power balances at the nodes is set as the objective function, the load flow may be formulated as

$P_{i}+j Q_{i}=V_{i} \cdot e^{j \delta_{i}} \sum Y_{i m} \cdot e^{-j \theta_{m}} \cdot V_{m} \cdot e^{-j \delta_{m}}$

Where,

$V_{i}, V_{m}:$ Voltage magnitude of $\mathrm{i}^{\text {th }}$ and $\mathrm{m}^{\text {th }}$ bus

$\delta_{i}, \delta_{m}$ : Phase angle of $\mathrm{i}^{\text {th }}$ and $\mathrm{m}^{\text {th }}$ bus

$Y_{i m}, \theta_{i m}$ : Admittance matrix element and its corresponding phase angle

$P_{i}, Q_{i}$ : Active and Reactive power of $\mathrm{i}^{\text {th }}$ bus

$\Delta P_{i}, \Delta Q_{i}$ : Active and Reactive power mismatches of the $\mathrm{i}^{\text {th }}$ bus

$\mathrm{SSE}=\sum_{i=1}^{n-1}\left(P_{s p}-P_{i}\right)^{2}+\sum_{i=1}^{l}\left(Q_{s p}-Q_{i}\right)^{2}$

$\max \_\mathrm{E}=\max \left(\left[\left.\left(P_{s p}-P_{i}\right)\right|_{i=1 \ldots(n-1)},\left.\left(Q_{s p}-Q_{i}\right)\right|_{i=1 \ldots l}\right]\right)$

Subject to,

$Q_{\text {gen }, i}^{\min } \leq Q_{i} \leq Q_{\text {gen }, i}^{\max } ; \mathrm{i}=1, \ldots \mathrm{g}$

$V_{i}^{\min } \leq V_{i} \leq V_{i}^{\max } \& \delta_{i}^{\min } \leq \delta_{i} \leq \delta_{i}^{\max } ; \mathrm{i}=1, \ldots$ (n-1)

'SSE' and 'max_E' represent sum of the squares of errors and maximum error of the total power mismatches respectively. In the above $\mathrm{g}$ is the number of generator buses except the slack bus, 1 is the number of load buses, and $\mathrm{n}$ is the total number of buses in the system. $P, Q, V$ and $\delta$ represent active power, reactive power, voltage magnitude and phase angle respectively. Subscripts 'sp' represents the specified value and 'gen' represents the quantity associated with the generator bus. Superscripts ' $\min$ ' and 'max' are used to indicate the corresponding minimum and maximum limits. It may be noted here that when load flow problem is solved using conventional approach the constraint given in (5) becomes redundant, as the flat start is the accepted starting values of the load flow iterative solutions. In the non-conventional approach during random initialization, flat start cannot be adhered to. Generally max_E is reduced in each iteration for the desired convergence. In meta-heuristic / evolutionary methods, SSE is the limiting constrain for achieving convergence. In the proposed method, if the maximum error (Max_E) of the best solution is less than the specified tolerance i.e. 0.001 , the solution is converged.

\section{Fuzzy Load Flow}

The Fuzzy load flow algorithm (FLF) has been built up considering the decoupling properties of the power flow variables. The voltage magnitudes $(\mathrm{V})$ are coupled with the reactive power mismatches $(\mathrm{Q})$ considering the phase angles $(\delta)$ constants whereas the phase angles are dependent on the active power mismatches (P) keeping voltage magnitudes fixed. The popular fast decoupled load flow (FDLF) can be written as

$[\Delta P / V]=\left[B^{\prime}\right][\Delta \delta]$

$[\Delta \mathrm{Q} / \mathrm{V}]=[\mathrm{B} "][\Delta \mathrm{V}]$

Here $B^{\prime}$ and $B^{\prime \prime}$ are the constant sparse matrices. Constant sparse matrix can be calculated in different ways. But, for the proposed decoupled fuzzy load flow, $B^{\prime}$ and $B^{\prime \prime}$ are determined as follows:

$$
B_{i i}^{\prime}=\sum_{k \in l} \frac{1}{X_{i k}}
$$




$$
\begin{aligned}
B_{i i}^{\prime \prime} & =\sum_{k \in l}\left(\frac{X_{i k}}{R_{i k}^{2}+X_{i k}^{2}}+Y_{c}\right) \frac{1}{a^{2}} ; \text { if tap is on side i } \\
& =\sum_{k \in l}\left(\frac{X_{i k}}{R_{i k}^{2}+X_{i k}^{2}}+Y_{c}\right) ; \text { if tap is on side } \mathrm{k}
\end{aligned}
$$

Where,

$\mathrm{Y}_{\mathrm{c}}$ : half line charges

$\mathrm{R}_{\mathrm{ik}}$ : line resistance between $\mathrm{i}$ and $\mathrm{k}$

$\mathrm{X}_{\mathrm{ik}}$ : line reactance between $\mathrm{i}$ and $\mathrm{k}$

$1:$ actual number of lines which are directly connected to the $\mathrm{i}^{\text {th }}$ bus

a : transformer ratio

The sparse matrices are determined only once before the starting of iteration. The diagonals of the sparse matrices are used to have an estimate of the correction element of the power flow variables. Considering a linear relationship between error and correction vector, it can be depicted that maximum correction vector $(\mathrm{CV})$ corresponds to maximum error and individual $\mathrm{CV}$ depends on its respective error (Vlachogiannis, 2001) i.e. the scale of CV depends on the magnitude of the corresponding error. To develop the Fuzzy rule, this philosophy is taken and it is considered as first criteria.

In the proposed decoupled FLF algorithm, another parameter, summation of error is also considered. From different types of modified FDLF, modified NRLF like Continuation Power Flow (CPF) (Ajjarapu et al, 1992), it is noticed that summation of error and its sign is also a decisive factor for the correction vector. Let the summation of error is large and its sign is negative. If individual error is negative, then definitely, the CV will be negative. On other case, if the individual error is positive, then the magnitude of $\mathrm{CV}$ is generally small and it may be positive or negative depending on the first criteria.

So, it is evident that in the proposed algorithm, the inputs for the $\mathrm{P}-\delta$ loop fuzzy system are active power mismatch $(\Delta \mathrm{P})$ and sum of active power mismatch $(\Sigma \Delta \mathrm{P})$ and the output is correction of phase angle $(\Delta \delta)$. Similarly the inputs for the $\mathrm{Q}-\mathrm{V}$ loop fuzzy system are reactive power mismatch $(\Delta \mathrm{Q})$ and sum of reactive power mismatch $(\Sigma \Delta \mathrm{Q})$ and the output is correction of voltage magnitude $(\Delta V)$. The system modeling is described below.

\subsection{Maximum range selection of input and output variables:}

The maximum ranges for the input and output variables of the fuzzy systems can be found by using the following formulae.

\subsubsection{For P- $\delta$ loop:}

$\Delta \delta_{\max }=\left[B_{m m}^{\prime}\right]^{-1}\left[\frac{\Delta P_{\max }}{V_{m}}\right]$

where $\Delta P_{\max }$ corresponds to $\mathrm{m}^{\text {th }}$ bus

$$
\begin{aligned}
\max \sum \Delta P & =\left|\Delta P_{\max }\right| ; \text { if }\left|\Delta P_{\max }\right|>\left|\sum_{i \in(n-1)} \Delta P_{i}\right| \\
& =\left|\sum_{i \in(n-1)} \Delta P_{i}\right| ; \text { otherwise }
\end{aligned}
$$

3.1.2 For $Q-V$ loop:

$\Delta V_{\max }=\left[B_{n n}\right]^{-1}\left[\frac{\Delta Q_{\max }}{V_{n}}\right]$

Where $\Delta Q_{\max }$ corresponds to $\mathrm{n}^{\text {th }}$ bus 


$$
\begin{aligned}
\max \sum \Delta Q & =\left|\Delta Q_{\max }\right| ; \text { if }\left|\Delta Q_{\max }\right|>\left|\sum_{i \in(n-2)} \Delta Q_{i}\right| \\
& =\left|\sum_{i \in(n-2)} \Delta Q_{i}\right| ; \text { otherwise }
\end{aligned}
$$

\subsection{Membership functions for input and output variables:}

Each variable (either input or output) is characterized by five membership functions in its universe of discourse. The name of the membership functions are given as follows:

NB : Negative big

NM : Negative medium

ZE : Zero

PM : Positive medium

PB : Positive Big

\subsubsection{For P- $\delta$ loop:}

The membership functions for the input and output variables of the P- $\delta$ loop of the proposed method are as shown in Figure 1, 2 and 3 .

\subsubsection{For Q-Vloop:}

We shall have three exactly similar figures for the membership functions of the input and output variables of the Q-V loop.

\subsection{Fuzzy rules:}

With the above membership functions of the input and the output variables, we can have 25 fuzzy rules for each of the loops.

\subsubsection{For P- $\delta$ loop:}

For the $\mathrm{P}-\delta$ loop the fuzzy rule base is shown in Table 1.For example, one linguistic rule of the P- $\delta$ loop may be written as

\section{Rule14: If $\Delta P$ is $\mathrm{PM}$ and $\sum \Delta P$ is $\mathrm{NM}$, then $\Delta \delta$ is $\mathrm{PM}$}

\subsubsection{For Q-Vloop:}

For the Q-V loop we shall get an exactly similar fuzzy rule base.

\subsection{Updating the power flow variables:}

The correction vectors are calculated by the fuzzy rules. Now the power flow variables are updated as

$V_{k}^{g^{\prime}}=V_{k}^{g}+\Delta V_{k}$

$\delta_{k}^{g^{\prime}}=\delta_{k}^{g}+\Delta \delta_{k}$

Where, $V_{k}^{g}$ and $\delta_{k}^{g}$ are the $\mathrm{k}^{\text {th }}$ voltage magnitude and phase angles at $\mathrm{g}^{\text {th }}$ generation respectively. Similarly, $V_{k}^{g^{\prime}}$ and $\delta_{k}^{g^{\prime}}$ are the updated voltage magnitudes and phase angle for the $\mathrm{k}^{\text {th }}$ node. The active and reactive power mismatches are calculated for the updated power flow variables. The sum of the squares of Error (SSE) and maximum power mismatch (max_E) are also determined.

Table 1. Fuzzy rule base for $\mathrm{P}-\delta$ loop

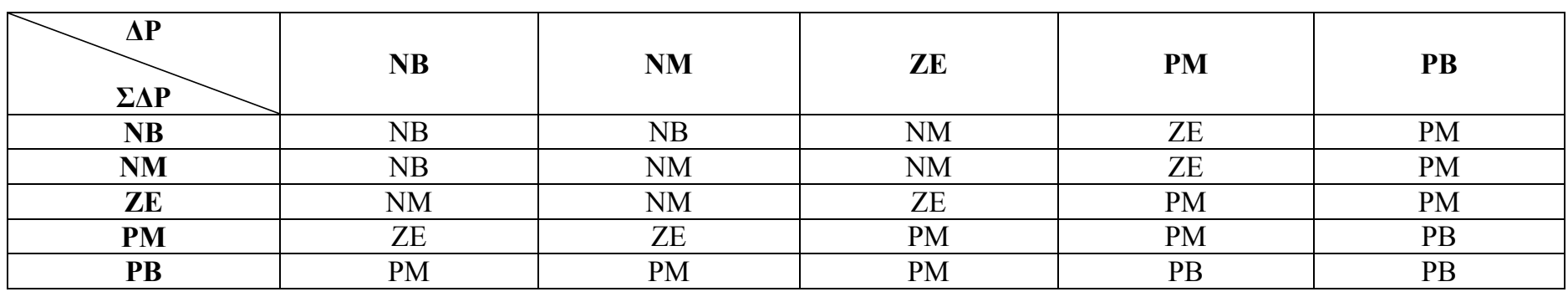




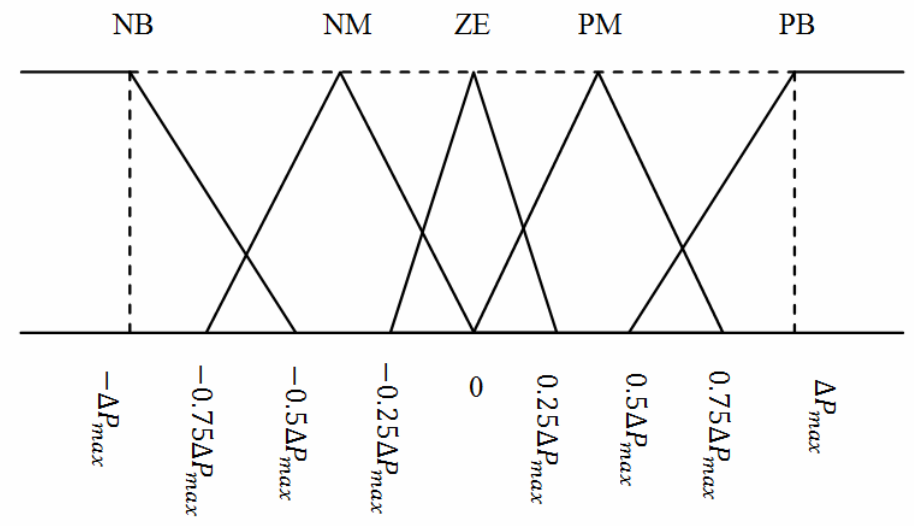

Figure 1. Membership function for input-1: active power mismatch $\Delta P$

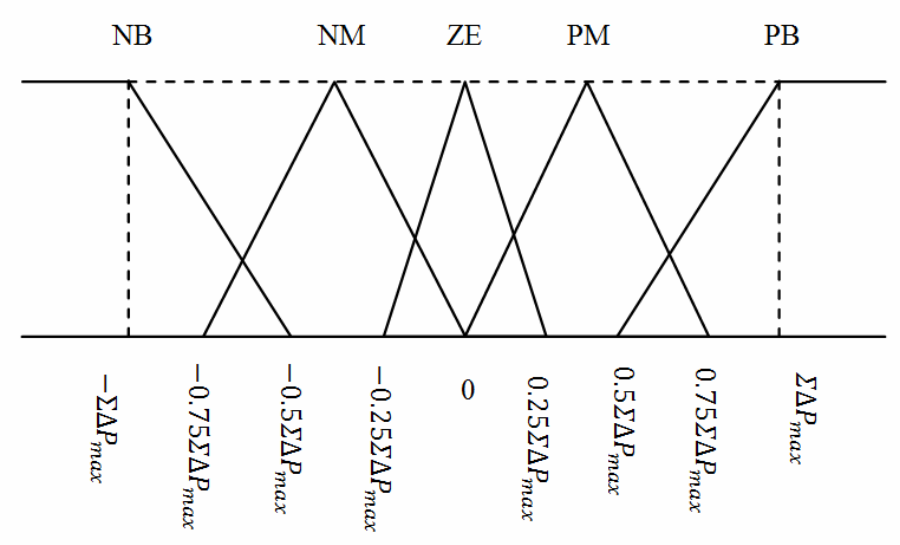

Figure 2. Membership function for input-2: sum of active power mismatch $\sum \Delta P$

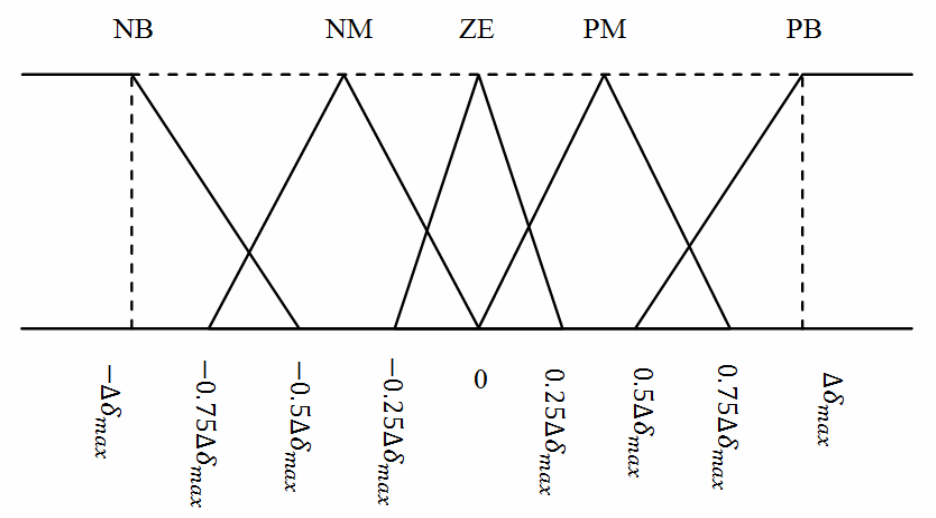

Figure 3. Membership function for output: phase angle correction vector $\Delta \delta$

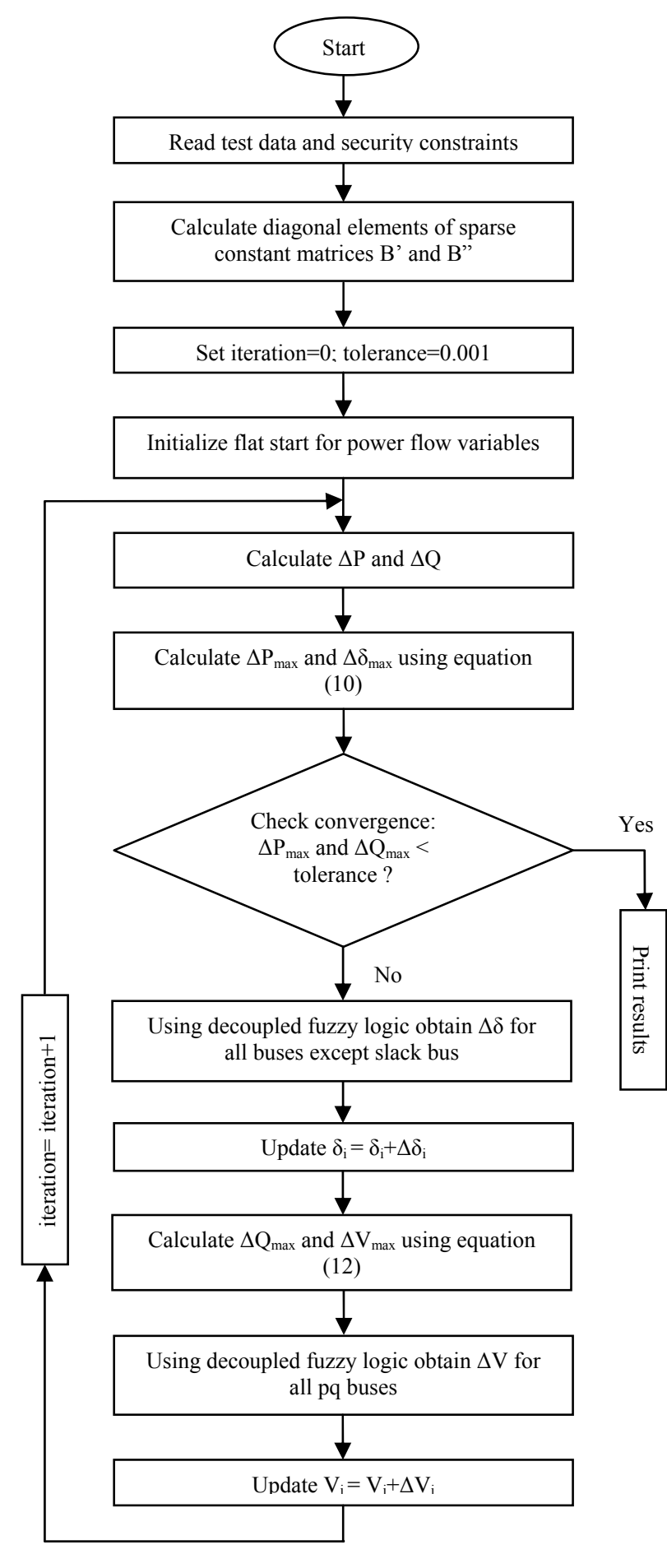

Figure 4. Flow chart for proposed algorithm

\subsection{Algorithm:}

The whole procedure of the proposed algorithm is shown by a flow chart in Figure 4. 


\section{Results}

The proposed algorithm is tested on different standard IEEE test systems such as 14, 30, 57 and 118 Bus. The presented algorithm has been developed and run on Pentium 4, 2.4 GHz, 256 MB RAM, 40 GB of Hard disc computer with the environment of MATLAB 2010.

Though the proposed algorithm takes more number of generations than conventional approaches, it provides better performance under stressed conditions. The Newton-Raphson load flow (NRLF) and Fast Decoupled load flow (FDLF) suffer from singularity problem at or near the high $\mathrm{R} / \mathrm{X}$ ratios and maximum loadability conditions. Better performance can be observed for optimal multiplier power flow (OMPF) method for ill-conditioned system, but the proposed load flow converges when all other conventional methods fail and also it provides robust performance for ill-conditioned systems. In order to show this, ill conditionings in the test systems have been introduced in two ways: by changing the $\mathrm{R} / \mathrm{X}$ ratio and by increasing the system loading. Comparison between the proposed algorithm and conventional methods in the context of loadability limits is given in Table 2. In this table only P, Q loads are multiplied and the generation settings are not modified for such increased loading conditions. It is assumed that all the additional power demands will be supplied by the slack generator.

$\mathrm{P}-\mathrm{V}$ or Q-V curve can be obtained using the proposed power flow algorithm. Figure 5 shows the P-V curve of IEEE 57 bus and solution of IEEE 57-bus test case under maximum loadability limit is given in Table 3 for evidence for the proposed algorithm. From Tables and Figure 5, it can be concluded that the performance of the proposed power flow algorithm regarding the loadability limits is better than other methods.

Line reactance are decreased to get high $\mathrm{R} / \mathrm{X}$ ratios and also on the other hand line resistance are increased in order to increase the line $\mathrm{R} / \mathrm{X}$ ratios as in (Amerongan, 1989). Table 4 and Table 5 show the test results for such cases when the conventional and proposed method just fail to give convergence. The case when the linear perturbation based power flow also fails represents the situations having no solution. From Tables, it is observed that the performances of the proposed method are better than the popular methods under critical conditions.

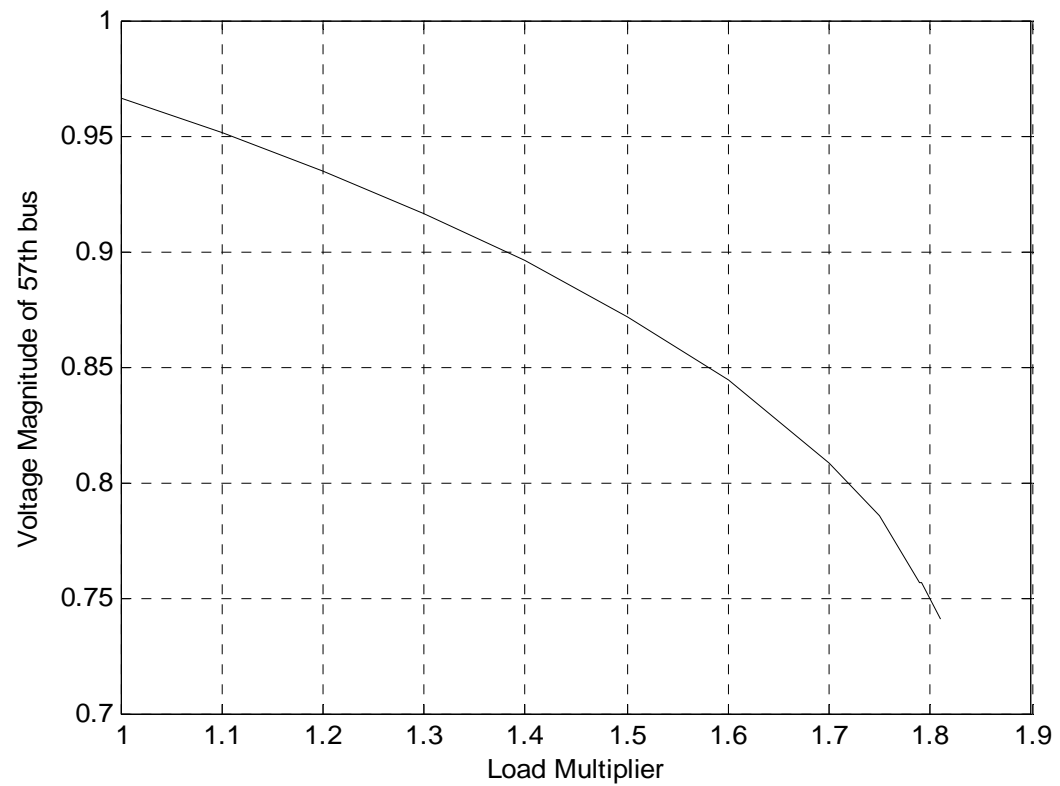

Figure 5. Variation of voltage magnitude of 57th node with load multiplier for IEEE 57-bus test system

Table 2. Load multiplier For Maximum loadability limits Of the different methods

\begin{tabular}{|c|c|c|c|c|}
\hline \multirow{2}{*}{ Test Systems } & \multicolumn{4}{|c|}{ Solution methods } \\
\cline { 2 - 5 } & FDLF & NRLF & $\begin{array}{c}\text { Optimal Multiplier } \\
\text { method }\end{array}$ & $\begin{array}{c}\text { Proposed } \\
\text { algorithm }\end{array}$ \\
\hline IEEE 14 bus & 4.0072 & 4.0078 & 4.0080 & 4.0115 \\
\hline IEEE 30 bus & 3.0156 & 3.0523 & 3.0527 & 3.0585 \\
\hline IEEE 57 bus & 1.7904 & 1.7916 & 1.7920 & 1.8003 \\
\hline IEEE 118 bus & 2.0373 & 2.0392 & 2.0392 & 2.0469 \\
\hline
\end{tabular}


Table 3. Line resistance multiplier for the critical R/X situations

\begin{tabular}{|c|c|c|c|c|}
\hline \multirow{2}{*}{ Test Systems } & \multicolumn{4}{|c|}{ Solution methods } \\
\cline { 2 - 5 } & FDLF & NRLF & $\begin{array}{c}\text { Optimal Multiplier } \\
\text { method }\end{array}$ & $\begin{array}{c}\text { Proposed } \\
\text { algorithm }\end{array}$ \\
\hline IEEE 14 bus & 4.4218 & 4.4223 & 4.4225 & 4.4291 \\
\hline IEEE 30 bus & 5.1194 & 5.1212 & 5.1215 & 5.1284 \\
\hline IEEE 57 bus & 4.4095 & 4.4102 & 4.4102 & 4.4152 \\
\hline IEEE 118 bus & 5.0690 & 5.0705 & 5.0705 & 5.0732 \\
\hline
\end{tabular}

Table 4. Line reactance multiplier for the critical R/X situations

\begin{tabular}{|c|c|c|c|c|}
\hline \multirow{2}{*}{ Test Systems } & \multicolumn{4}{|c|}{ Solution methods } \\
\cline { 2 - 5 } & FDLF & NRLF & $\begin{array}{c}\text { Optimal Multiplier } \\
\text { method }\end{array}$ & $\begin{array}{c}\text { Proposed } \\
\text { algorithm }\end{array}$ \\
\hline IEEE 14 bus & 0.0495 & 0.0491 & 0.0490 & 0.0419 \\
\hline IEEE 30 bus & 0.0558 & 0.0554 & 0.0552 & 0.0401 \\
\hline IEEE 57 bus & 0.0668 & 0.0655 & 0.0655 & 0.0588 \\
\hline IEEE 118 bus & 0.0714 & 0.0696 & 0.0696 & 0.0624 \\
\hline
\end{tabular}

\section{Conclusions}

The decoupling properties of power systems are used to develop fuzzy load flow. Two separate loops such as phase angle \& active power and voltage magnitude \& reactive power are operated one after another in a single iteration. Maximum power mismatches and summation of error are two input ranges. To obtain correction vector, 25 fuzzy rules are created. As there is no derivative/differentiation, the proposed algorithm performs better than conventional approaches under critical conditions such as high $\mathrm{R} / \mathrm{X}$ ratio, high loadability etc.

\section{Acknowledgement}

The financial support by Department of Science and Technology, New Delhi vide project number SR/FT/ET-057/2008 is gratefully acknowledged.

\section{References}

Ajjarapu V., and C. Christy, "The Continuation Power Flow: A Tool For Steady State Voltage Stability Analysis", IEEE Trans. Power Syst. PS-7 (1992) 416-423.

Dragoslav R., Anjan Bose, "A Modification to the Fast Decoupled Power Flow For Networks with High R/X Ratios", IEEE Transactions on Power Systems, Vol. 3, No. 2, May 1988.

Iwamoto S., and Tamura Y., "A Load Flow Calculation Method For Ill-conditioned Power System”, IEEE Transaction on Power Apparatus, PAS-100, Vol., No. 4, April 1981.

Lo K.L., Lin Y.J., Trecat J. and Crappe M., "Improvement of FACTS incorporated power systems stability performance through fuzzy control". Proceedings of 3rd TPEC conference, Singapore, 1997, pp. 16\&165.

Lo K.L., Lin Y.J. and Siew W.H., "Fuzzy-logic method for adjustment of variable parameters in load-flow calculation", IEE Proc.-Gener. Trunsni. Distrib., 1999, 146, (3), pp. 276-282.

Lu Wang, X. Rong Li, "Robust Fast Decoupled Power Flow”, IEEF Transactions on Power Systems, Vol. 15, No. I, February 2000.

Miranda V. and Matos M., "Distribution system planning with fuzzy models and techniques", Proceedings of CIRED89, Bristol, $\mathrm{UK}, 1998$.

Tinney W.F., and Hart C.E., "Power Flow Solution by Newton's method", IEEE Trans. Power Apparatus \& Systems, Vol. PAS -86, pp. 1449-1456, Nov. 1967.

Van Amerongan R. A. M., “A General Purpose Version of the Fast Decoupled Load Flow”, IEEE Trans. Power System, Vol. 4,pp. 760-770, May 1989.

Vlachogiannis J.G., Fuzzy logic application in load flow studies", IEE Proceedings on Generation, Transmission and Distribution,

Vol. 148 No. 1 January, 2001.

Wong K. P., Yuryevich J., and Li A., “ Evolutionary Programming Based Load Flow Algorithm For Systems Containing Unified 
Power Flow Controllers", IEE Proc.-Gener. Transm. Distrib., Vol. 150 No. 4, pp. 441-446, July 2003.

Wong K. P., Li A., and Law M.Y., “ Development of Constrained Genetic Algorithm Load Flow Method”, IEE Proc.-Gener. Transm. Distrib., Vol. 144, No. 2, pp. 91-99, March 1997.

Wong K. P., Li A., and Law M.Y., “ Advanced Constrained Genetic Algorithm Load Flow Method”, IEE Proc.-Gener. Transm. Distrib., Vol. 140 No. 6 , pp. 609-616, November 1999.

\section{Biographical notes}

Dr. Parimal Acharjee passed B.E.E. from North Bengal University \& completed M.E.E. and Ph. D. from Jadavpur University, Kolkata, India. After three year industrial experience, he continued his teaching profession in MCKVIE, Howrah, BPPIMT, Kolkata, NIT Silchar, India. He is presently working in NIT Durgapur, India as an Associate Professor. He received research fund for Young Scientist under 'Fast Track scheme' by DST, New Delhi. His current research interest is the application of soft-computing techniques in various power system problems.

Kawsar Ali is pursuing his B. Tech. degree in Electrical Engineering in National Institute of Technology, Durgapur, India. He is currently in his final semester. His current research interest is the application of soft-computing techniques in various power system problems.

Received January 2011

Accepted March 2011

Final acceptance in revised form April 2011 